\title{
Percepción de funcionalidad familiar en adolescentes escolarizados en instituciones educativas públicas de Medellín (Colombia), 2014
}

\section{Perception of Family Functioning in Adolescents enrolled in Public Education Institutions in Medellín (Colombia), 2014}

\author{
Luis Felipe Higuita-Gutiérrez ${ }^{1} \mathrm{ORCID}$ - Jaiberth Antonio Cardona-Arias ${ }^{2 \mathrm{ORCID}}$ \\ 1,2. Universidad de Antioquia \\ Colombia
}

Fecha correspondencia:

Recibido: diciembre 15 de 2015.

Aceptado: julio 19 de 2016.

Forma de citar:

Higuita, L.F., \& Cardona, J. (2016).

Percepción de funcionalidad

familiar en adolescentes

escolarizados en instituciones

educativas públicas de Medellín

(Colombia), 2014. Rev. CES Psicol.,

9(2), 167-178.

Open access

(c) Copyright

Licencia creative commons

Etica de publicaciones

Revisión por pares

Gestión por Open Journal System

ISSN: 2011-3080

DOI: http://dx.doi.org/10.21615/

cesp.9.2.11

Sobre los autores:

1. MSc en Educación y desarrollo humano. Docente Escuela de Microbiología, Universidad de Antioquia.

Comparte

f06.

\section{Resumen}

La composición y funcionalidad de la familia son la base estructurante para la construcción de los comportamientos en los adolescentes; en esta medida se aboga por mantener una buena funcionalidad familiar. En Medellín son pocos los estudios sobre esta problemática, que permitan identificar subgrupos de riesgo, focalizar necesidades y diseñar estrategias de atención, prevención y promoción. El objetivo de este estudio fue analizar la percepción de funcionalidad familiar, según factores sociodemográficos, en adolescentes de la ciudad de Medellín. Para ello se realizó un estudio analítico en 3460 adolescentes seleccionados por muestreo probabilístico. Se evaluaron las propiedades psicométricas de la escala de funcionalidad familiar, se calcularon estadísticos descriptivos, pruebas de hipótesis y regresión multinomial. Los resultados sugieren una elevada prevalencia de disfuncionalidad familiar que se asocia con la edad, la escolaridad de los padres, el sector de residencia y el tipo de familia. Por otro lado, se puso en evidencia la disponibilidad de una escala válida y fiable para tamizar esta problemática y monitorizar su evolución en estudios posteriores.

Palabras clave: Funcionalidad familiar, Adolescentes, Colombia.

\section{Abstract}

Family functioning and structure are the basis for building behaviors in adolescents, in this sense, providing an appropriate family functioning is required. There are few studies in Medellin about this social problem that allow professionals in this field identifying the risky factors, targeting the needs and designing strategies for assistance, prevention and promotion. The purpose of this paper was to study family functioning perception of adolescents in Medellín-Colombia, according to sociodemographic factors. An analytical study was conducted in 3.460 adolescents selected by probability sampling. Descriptive statistics, hypothesis testing and multinomial regression were estimated. It was found a high prevalence of family dysfunction associated with age, parental education, residence zone and family type. On the other hand, it was evident the availability of a valid and reliable scale to weigh up this issue and monitor its evolution in subsequent studies.

Keywords: Family functioning, Adolescents, Colombia. 
Pág 168

2. MSc en Epidemiología.

Docente Escuela de

Microbiología, Universidad

de Antioquia, Facultad

de Medicina Universidad

Cooperativa de Colombia.

\section{Introducción}

La familia es una institución que desempeña una función privilegiada en la sociedad, al ejercer las influencias más tempranas, directas y duraderas en la formación de los individuos. Esta importancia se debe, entre otras cosas, a que en ella se presenta la transmisión de creencias, valores y pautas de comportamiento de una generación a otra (American Academy of Pediatrics, 2003). Uno de los momentos trascendentales al interior de las familias es la adolescencia de los hijos, debido a que en esta etapa de la vida de ellos se presentan cambios físicos, psíquicos y sociales que condicionan aspectos como la actitud de rechazo hacia las normas, el manejo de la autoridad, las fluctuaciones emocionales y se cuestionan las costumbres, valores y normas introyectadas hasta el momento (Uparela et al, 2011).

Teniendo en cuenta lo anterior, se ha sugerido que la composición y funcionalidad de la familia son la base estructurante para la construcción de los comportamientos en los adolescentes, y en esta medida se han presentado estudios que abogan por mantener una buena funcionalidad familiar (Araujo et al, 2012). Se entiende por familia funcional aquella en la que se estimula la capacidad para resolver unidos las crisis que se presentan al interior y al exterior de la misma, en la que además se expresan afectos y apoyo, se permite el crecimiento y desarrollo de cada integrante y se genera interacción entre ellos, respetando la autonomía y el espacio individual (Muyibi, Ajayi, Irabor, \& Ladipo, 2010; González, Gimeno, Meléndez, \& Córdoba, 2012).

En contraposición a la funcionalidad familiar, estudios previos han descrito que adolescentes con familias disfuncionales presentan conductas como bajo rendimiento académico (Moreno \& Chauta, 2012), consumo de sustancias psicoactivas (Ashby \& Yaeger, 2003), pobres relaciones sociales (Bell \& Bell, 1982), síntomas depresivos (Culpin, Heron, Araya, Melotti, \& Joinson, 2013), impulsividad (Negrete \& Vite, 2011) e intento de suicidio (Guibert \& Torres, 2001). De la misma manera, se ha reportado que aspectos como la composición de la familia, el nivel educativo, el sexo y el estrato socioeconómico de los padres, son factores que influyen en el funcionamiento de las familias (Palomar \& Márquez, 1999; Fondo de las Naciones Unidas para la Infancia [UNICEF], 2003).

Por consiguiente, la funcionalidad familiar se ha constituido en un tema de gran relevancia para los investigadores sociales y para la salud pública, por lo que se han reportado múltiples investigaciones para dar cuenta de ella. De esta manera, en Perú se han reportado prevalencias de disfuncionalidad familiar del 35\% (Cuba, Jurado, Romero, \& Cuba, 2013), en Costa Rica del 31,4\% (Ulate-Gómez, 2013) y en Brasil del 26,8\% (Araujo et al., 2012). En Colombia se han realizado múltiples estudios, uno de ellos, realizado en Bogotá, informó una prevalencia de disfuncionalidad familiar del $59 \%$ (Moreno \& Chauta, 2012), mientras que otro realizado en Cartagena informó una prevalencia del 51,8\% (Gómez-Bustamante, Castillo-Ávila, \& Cogollo, 2013). En Medellín se han realizado estudios de funcionalidad familiar en niños con diagnóstico de asma, en los que se encontró que el $77,3 \%$ de los niños que tienen asma no controlada presentan disfunción familiar (Berbesí, García, Segura, \& Posada, 2013). De la misma manera, la funcionalidad familiar se ha estudiado en adolescentes con riesgo de suicidio, en quienes se reportó mayor frecuencia de familias disfuncionales (Alcaldía de Medellín, 2012).

No obstante, los estudios descritos en el párrafo previo presentan algunas limitaciones que impiden la inferencia o extrapolación de sus resultados a otras poblaciones, como circunscribirse a individuos enfermos, ausencia de evaluación de las propie- 
Pág 169

Es importante que los adolescentes participen del bienestar de sus familias, de ahí, que las políticas sociales dirigidas a esta población deben trascender el enfoque tradicional centrado en el individuo y abordar la familia en conjunto (Cuba et al., 2013). dades psicométricas de los instrumentos utilizados para la tamización de la disfuncionalidad y falta de descripción de la funcionalidad familiar desde la percepción de los adolescentes.

Por otra parte, Medellín presenta unas características especiales relacionadas con la distribución en comunas, al interior de las cuales existen diferencias económicas y culturales que suponen variaciones en el perfil de la funcionalidad familiar y que no han sido investigadas; prueba de ello es que en la literatura científica no se dispone de un estudio sobre la funcionalidad familiar en esta ciudad, cuya tamización se haya desarrollado con instrumentos validados para captar diferencias según las comunas o zonas de ésta.

Un estudio que incluya la población adolescente de la ciudad, aborde la funcionalidad familiar con instrumentos validados, tenga en cuenta aspectos centrales como la composición de la familia y evalúe las diferencias en relación con características sociodemográficas, es de gran relevancia, en cuanto permite identificar subgrupos de riesgo, focalizar necesidades y diseñar estrategias de atención y promoción de la salud y prevención de la enfermedad.

Además, es importante que los adolescentes participen del bienestar de sus familias, de ahí, que las políticas sociales dirigidas a esta población deben trascender el enfoque tradicional centrado en el individuo y abordar la familia en conjunto (Cuba et al., 2013). En esta medida, la investigación sobre funcionalidad familiar posibilita la orientación del diseño de políticas públicas intersectoriales, que conciban la misma como red de apoyo primario para el cuidado de los adolescentes, en conjunción con el contexto académico, la asistencia sanitaria y la educación.

Teniendo en cuenta lo anterior, se diseñó este estudio con el objetivo de analizar la funcionalidad familiar según factores sociodemográficos, en adolescentes escolarizados de la ciudad de Medellín, en el año 2014.

\section{Materiales y métodos}

\section{Tipo de estudio}

Transversal analítico.

\section{Sujetos de estudio}

La población de estudio estuvo conformada por los estudiantes de décimo y undécimo grado, matriculados durante el año 2014 en las instituciones de educación pública de Medellín. La muestra fue de 3.460 estudiantes de 18 instituciones educativas y se calculó teniendo en cuenta una población de 200.000 sujetos, una confianza del $95 \%$, una proporción de disfuncionalidad familiar del 50\%, una precisión de muestreo del $2 \%$ y una corrección de muestreo del 15\%. Cabe aclarar que en la zona que agrupa los corregimientos de la ciudad, sólo se debería muestrear una institución educativa según el muestreo estratificado; sin embargo, a la muestra final se sumaron los estudiantes de tres corregimientos, dado que éstos presentaban una alta variabilidad socioeconómica, demográfica y cultural entre sí.

La selección de las 18 instituciones se hizo de manera aleatoria y proporcional al total de instituciones educativas de cada una de las siete zonas en las que se divide la ciudad; seis urbanas (Nororiental, Noroccidental, Centroriental, Centroccidental, 
Suroriental y Suroccidental) y una rural llamada "Corregimientos". En estos colegios, se encuestó la totalidad de los estudiantes de los grados $10^{\circ}$ y $11^{\circ}$ y se excluyeron quienes presentaran alteraciones cognitivas según el criterio del psicólogo o el profesor de cada institución, las personas que rechazaron la participación y quienes solicitaron remuneración.

\section{Instrumentos de recolección}

Escala de funcionalidad familiar. Para la recolección de la información, se utilizó la escala de funcionalidad familiar APGAR (Por su significado en inglés: Adaptation, Partnership, Growth, Affection, Resolve). Este instrumento, disponible en Chino, Inglés, Portugués y Español, se utiliza como prueba de tamización y permite que las personas manifiesten el grado de satisfacción con la funcionalidad de su familia, a partir de cinco ítems: la adaptabilidad, entendida como el grado de satisfacción con la ayuda o el apoyo recibido, para resolver los problemas en momentos en los que el equilibrio de la familia se ve amenazado por un factor de estrés; la cooperación, que se define como la participación en la toma de decisiones y la comunicación mutua e indica el grado de poder al interior de la familia; el desarrollo, que indica la satisfacción de los miembros de la familia frente a las posibilidades de maduración emocional, física y de autorrealización al interior de la familia; la afectividad, que es la manera en la que se comparten las experiencias emocionales, las relaciones de afecto y atención entre los miembros de la familia, y la capacidad resolutiva, entendida como la satisfacción con el tiempo, el espacio y el dinero que los miembros de la familia dedican para compartir entre ellos. Este instrumento genera un puntaje de 0 a 10: los resultados de cero a tres indican disfuncionalidad grave, de cuatro a seis, disfuncionalidad moderada y de siete a diez, funcionalidad familiar (Gómez \& Ponce, 2010).

\section{Procedimiento}

Para la recolección de la información se contactó a los rectores de las instituciones educativas y, a través de un profesor delegado por Institución, se presentó el proyecto a los estudiantes quienes avalaron su participación voluntaria en el estudio y diligenciaron una encuesta anónima que, además del APGAR, contenía información relacionada con el tipo de familia, el estado de salud y características sociodemográficas como el lugar de residencia, el sexo, la edad del estudiante, la edad y escolaridad de los padres y el estrato socioeconómico.

Durante la recolección de la información se controlaron sesgos en el encuestador, en el instrumento y en el encuestado. El encuestador realizó un entrenamiento, que incluyó un protocolo con las definiciones operativas de las variables y directrices sobre el trabajo de campo; al instrumento se le aplicó una versión validada para la población de estudio; al encuestado se le garantizó la confidencialidad, el anonimato y se excluyeron los participantes que pudiesen generar sesgos de memoria por limitaciones cognitivas. Además, se realizó una prueba piloto en el 10\% de la muestra.

\section{Análisis de la información}

La evaluación psicométrica del APGAR se realizó con los criterios de consistencia interna, fiabilidad, validez de constructo y validez predictiva. Para evaluar la consistencia interna, se calcularon correlaciones de Pearson entre cada ítem y el puntaje global de funcionalidad familiar, y se tomaron como favorables valores superiores a 0,4. La fiabilidad se evaluó a través del coeficiente $\alpha$ de Cronbach, para el que se consideraron aceptables los valores superiores a 0,7. La validez de constructo se realizó con un análisis factorial exploratorio, de componentes principales para un 
Pág 171

En cuanto a la percepción de la funcionalidad familiar, se encontró que el 69,4\% ( $n=2405)$ de los adolescentes indicó formar parte de hogares funcionales mientras que $30,6 \%(n=1055)$ de integrar familias disfuncionales. De éstas, el 75,5\% ( $n=797)$ presentan disfuncionalidad moderada y el 24,5\% $(n=258)$ disfuncionalidad grave (Figura 2). factor (Funcionalidad familiar), se calcularon las cargas factoriales, aceptando valores superiores a 0,4. Como etapa complementaria a la validez de constructo, se contrastaron los puntajes de la escala entre poblaciones diversas (Sanos/Enfermos), a través de la prueba de comparación de proporciones con el Estadístico Z, para verificar la capacidad del instrumento de detectar las diferencias (Cardona-Arias, Caro-Londoño, González, \& Franco-Mosquera, 2014; Ramada-Rodilla, Serra-Pujadas \& Delclós-Clanchet, 2013). La validez predictiva se calculó a través del porcentaje de la varianza de la funcionalidad familiar que es explicada por los ítems que conforman el APGAR, considerando resultados aceptables por encima del 50\% (CardonaArias et al., 2014; Ramada-Rodilla et al., 2013).

Para describir la población de estudio, se calcularon frecuencias absolutas, proporciones con sus intervalos de confianza del $95 \%$ y medidas de resumen. El supuesto de normalidad se evaluó con la prueba Kolgomorov-Smirnov, con corrección de Lilliefors. Se realizó ANOVA de un factor, tras la evaluación del supuesto de homocedasticidad con el estadístico de Levene, para comparar la funcionalidad familiar con la edad de los adolescentes, la edad de los padres, el número de personas en el hogar y los años de estudio de los padres. Para comparar la funcionalidad familiar frente al sector de residencia, el grado, sexo, estrato social y tipo de familia, se realizó el Chi cuadrado de independencia. Para cuantificar la confusión, se realizó una regresión logística multinomial.

Los análisis se realizaron en SPSS $21.0^{\circledR}$ y en EPIDAT 3.1 con una significación estadística de 0,05.

\section{Aspectos éticos}

Se aplicaron los principios de confidencialidad, anonimato y los demás suscritos en la Declaración de Helsinki y la Resolución 8430 de 1993 del Ministerio de Salud de Colombia. Esta investigación se enmarca en el macroproyecto sobre Violencia escolar, funcionalidad familiar y calidad de vida en adolescentes escolarizados en instituciones públicas de Medellín, del año 2014, avalado por el Comité de Bioética de la Universidad Cooperativa de Colombia.

\section{Resultados}

Se halló una proporción similar de estudiantes en grado décimo, el 52,5\% (1815), y undécimo, el 47,5\% (1645). El 60,2\% (2083) fueron mujeres de estrato socioeconómico entre bajo y medio y el $18,5 \%$ refirió padecer alguna enfermedad (Figura 1). La

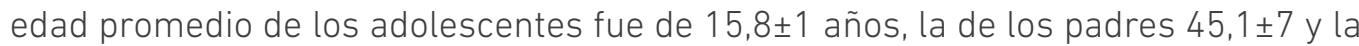
de las madres $41,4 \pm 6$ años. Los años de escolaridad promedio de los padres fueron de $8,71 \pm 3,3$ y de las madres $9,3 \pm 3,1$ (Figura 1).

En lo que concierne al tipo de familia, se destaca que el $45,5 \%$ de los adolescentes forman parte de familias monoparentales $(n=1404)$ y el $6,6 \%(n=205)$ refirió la ausencia de ambos padres. Entre las familias monoparentales, el 91,4\% reportó la ausencia del padre, mientras que el $8,6 \%$ refirió la ausencia de la madre. Se resalta que el $29,5 \%(n=1022)$ de los adolescentes conforman familias extensas, en tanto que el hogar es compartido con otras personas como abuelos, tíos y primos (Figura 2).

En cuanto a la percepción de la funcionalidad familiar, se encontró que el 69,4\% ( $n=2405)$ de los adolescentes indicó formar parte de hogares funcionales mientras que 30,6\% $(n=1055)$ de integrar familias disfuncionales. De éstas, el 75,5\% ( $n=797)$ presentan disfuncionalidad moderada y el 24,5\% ( $n=258$ ) disfuncionalidad grave (Figura 2). 


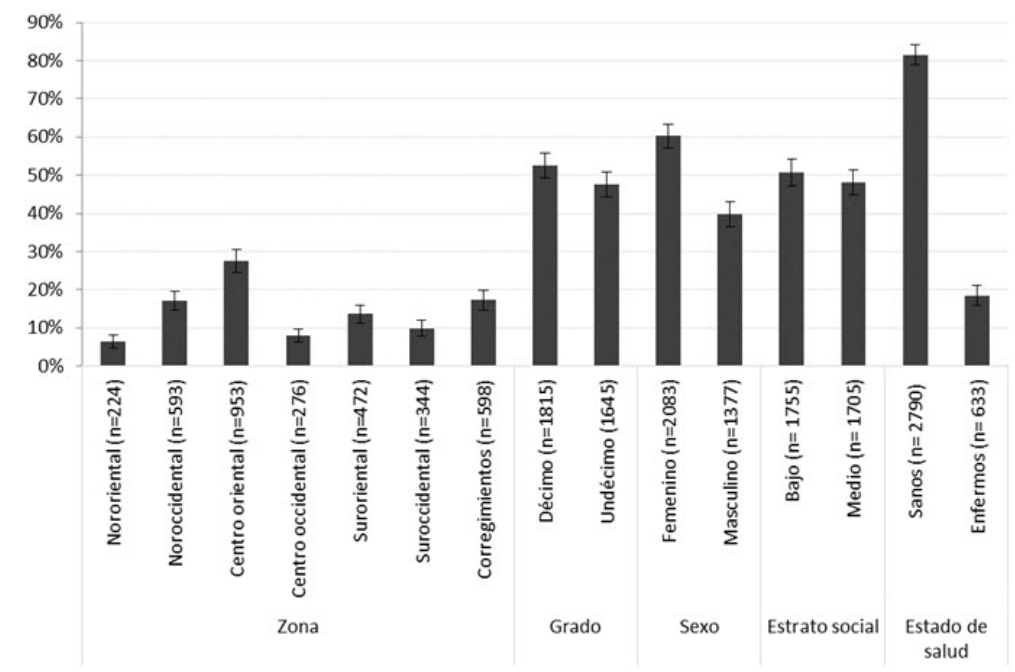

Figura 1. Características sociodemográficas del grupo de estudio

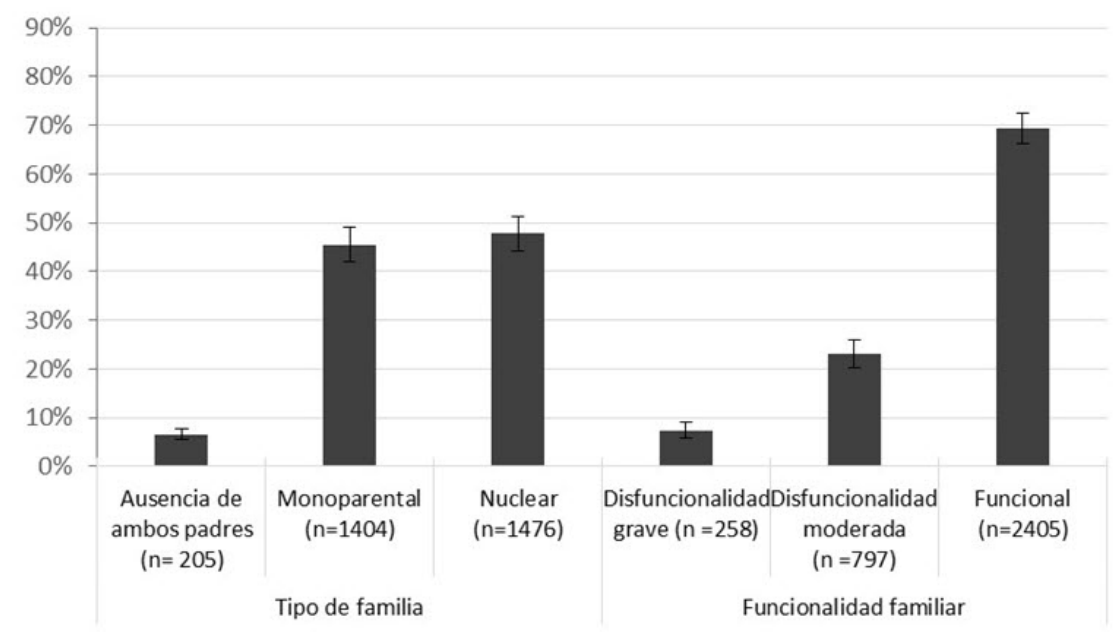

Q Figura 2. Descripción del tipo de familia y funcionalidad familiar

Previo a la evaluación de la funcionalidad familiar, se realizó la evaluación psicométrica de la escala APGAR en la población de referencia, la cual presentó un 100\% de éxito en la consistencia interna, ya que se observaron correlaciones mayores a 0,4 para cada uno de los ítems que la conforman, con el puntaje global de funcionalidad familiar. Además, presentó buena fiabilidad según el coeficiente $\alpha$ de Cronbach de 0,86 . Se observó excelente validez de constructo en tanto que los coeficientes Lambda entre cada ítem y el factor de "funcionalidad familiar" fueron superiores a 0,4. Adicionalmente, se confirmó que los cinco ítems representan un único factor (Funcionalidad familiar), que explica el $52,77 \%$ de la varianza obtenida en los puntajes del APGAR (Tabla 1).

Además, la prueba de comparación de proporciones evidenció diferencias estadísticas entre la percepción de funcionalidad familiar de adolescentes enfermos y sanos, con un Estadístico Z de 3,752 ( Vp=0,000) y un intervalo de confianza para la diferencia de proporciones entre el $3,5 \%$ y el $11,9 \%$. 
Tabla 1. Propiedades psicométricas de la escala APGAR: Consistencia interna, fiabilidad, validez de constructo y validez predictiva

\begin{tabular}{lcc}
\hline & Consistencia interna* & Coeficiente Lambda** \\
\hline Satisfecho con ayuda de la familia & 0,760 & 0,780 \\
Conversan los problemas en familia & 0,735 & 0,721 \\
Toman decisiones en conjunto & 0,729 & 0,707 \\
Satisfecho con el tiempo en familia & 0,715 & 0,700 \\
Siente que la familia lo quiere & 0,686 & 0,722 \\
\hline Validez predictiva & 52,8 & \\
(\% de la varianza explicada) & 0,86 & $70,9(69,2 ; 72,6)$ \\
\hline Fiabilidad ( $\alpha$ de Cronbach) & Sanos & $63,2(59,3 ; 67,0)$ \\
\hline Validez de constructo (\% familias funcionales & Enfermos & \\
\hline
\end{tabular}

Correlaciones de Pearson ítem-puntaje de la escala (todos presentaron valor $\mathrm{p}<0,01$ ).

${ }^{* *}$ Carga factorial: Coeficientes Lambda para cada ítem frente al factor construido.

Tabla 2. Análisis de la percepción de funcionalidad familiar según las características sociodemográficas del grupo de estudio

\begin{tabular}{|c|c|c|c|c|}
\hline & Grave & Moderada & Normal & Valor $p$ \\
\hline Edad $X \pm D E$ & $15,8(1,0)$ & $15,9(1,0)$ & $15,7(1,0)$ & $0,000^{1 * *}$ \\
\hline \# Personas Hogar $\mathrm{X} \pm \mathrm{DE}$ & $4,5(1,6)$ & $4,5(1,5)$ & $4,4(1,5)$ & $0,434^{1}$ \\
\hline Edad Padre $X \pm D E$ & $45,2(7,3)$ & $45,1(7,3)$ & $45,1(7,3)$ & $0,939^{1}$ \\
\hline Edad Madre $X \pm D E$ & $41,4(6,4)$ & $41,3(6,3)$ & $41,3(6,2)$ & $0,729^{t}$ \\
\hline Años de estudio padre $X \pm D E$ & $8,1(3,3)$ & $8,4(3,3)$ & $8,9(3,2)$ & $0,000^{1 *}$ \\
\hline Años de estudio madre $X \pm D E$ & $9,2(3,2)$ & $8,9(3,0)$ & $9,4(3,1)$ & $0,000^{1^{* *}}$ \\
\hline \multicolumn{5}{|l|}{ Sector \%(\#) } \\
\hline Nororiental & $9,4(21)$ & $30,4(68)$ & $60,3(135)$ & \\
\hline Noroccidental & $7,8(46)$ & $23,9(142)$ & $68,3(405)$ & \\
\hline Centro oriental & $8,0(76)$ & $23,7(226)$ & $68,3(651)$ & \\
\hline Centro occidental & $8,0(22)$ & $19,6(54)$ & $72,5(200)$ & \multirow{4}{*}{$0,038^{*}$} \\
\hline Suroriental & $7,6(36)$ & $20,3(96)$ & $72,0(340)$ & \\
\hline Suroccidental & $3,8(13)$ & $21,5(74)$ & $74,7(257)$ & \\
\hline Corregimientos & $7,4(44)$ & $22,9(137)$ & $69,7(417)$ & \\
\hline \multicolumn{5}{|l|}{ Grado } \\
\hline Décimo & $7,5(137)$ & $22,3(404)$ & $70,2(1274)$ & \multirow{2}{*}{$0,523^{\ddagger}$} \\
\hline Undécimo & $7,4(121)$ & $23,9(393)$ & $68,8(1131)$ & \\
\hline \multicolumn{5}{|l|}{ Sexo } \\
\hline Femenino & $8,0(166)$ & $23,9(497)$ & $68,2(1420)$ & \multirow{2}{*}{$0,094^{\ddagger}$} \\
\hline Masculino & $6,7(92)$ & $21,8(300)$ & $71,5(985)$ & \\
\hline \multicolumn{5}{|l|}{ Estrato social } \\
\hline Bajo & $7,6(133)$ & $23,6(414)$ & $68,8(1208)$ & \multirow{2}{*}{$0,676^{\ddagger}$} \\
\hline Medio & $7,3(125)$ & $22,5(383)$ & $70,2(1197)$ & \\
\hline \multicolumn{5}{|l|}{ Tipo de familia } \\
\hline Ausencia de ambos padres & $12,7(26)$ & $30,7(63)$ & $56,6(116)$ & \multirow{3}{*}{$0,000^{\ddagger^{* *}}$} \\
\hline Monoparental & $8,4(118)$ & $24,4(342)$ & $67,2(944)$ & \\
\hline Nuclear & $5,7(84)$ & $21,2(313)$ & $73,1(1079)$ & \\
\hline
\end{tabular}

${ }^{1}$ Anova de un factor. ${ }^{\ddagger}$ Chi cuadrado de independencia.

"El estadístico es significativo en el 0,05. "El estadístico es significativo en el 0,01. 
Pág 174

Las medidas de atención primaria, prevención y promoción de las buenas relaciones familiares deben ser dirigidas reconociendo las particularidades de cada zona.
Al analizar la relación entre las características sociodemográficas y el grado de percepción de funcionalidad familiar, se encontraron diferencias estadísticamente significativas con la edad, los años de estudio de los padres, el sector de la ciudad y el tipo de familia. Con base en estas variables asociadas se configura un perfil de riesgo para la disfuncionalidad familiar, en el que predominan los subgrupos de familias con adolescentes próximos a los 16 años, cuyos padres tienen una baja escolaridad, residentes en la zona norte de la ciudad y particularmente en la zona nororiental, así como familias en las que hay ausencia de al menos uno de los padres (Tabla 2). A partir de una regresión logística multinomial, se confirmó que las asociaciones bivariadas eran reales y no producto de una posible confusión, dado que todas las asociaciones estadísticas permanecieron luego del ajuste multivariante.

\section{Discusión}

La percepción de disfuncionalidad familiar en el grupo de estudio fue del 30,6\%, resultado coherente con lo descrito en investigaciones realizadas en Perú (Cuba et al., 2013), Costa Rica (Ulate-Gómez, 2013) y Brasil (Araujo et al., 2012); y divergente de estudios realizados en ciudades de Colombia como Bogotá (Moreno \& Chauta, 2012) y Cartagena, en las cuales se han reportado mayores prevalencias de percepción de disfuncionalidad familiar (Gómez-Bustamante et al., 2013). Estas diferencias podrían explicarse porque en la ciudad de Medellín se vienen implementando estrategias específicas dirigidas a la intervención familiar, como las consignadas en el protocolo de acompañamiento familiar del programa "Medellín Solidaria" (Alcaldía de Medellín, 2009), el programa "Buen vivir en familia" (Alcaldía de Medellín, 2014) y las actividades del Centro de familia de la Universidad Pontificia Bolivariana, las cuales aportan elementos importantes para el trabajo terapéutico y preventivo (Observatorio para la equidad y la integración social en Medellín y Antioquia, 2006). No obstante, también es posible que las diferencias se deban a características específicas de la población, al proceso de validación de las escalas o demás factores no explorados en esta investigación.

Además de lo anterior, en este estudio se encontraron diferencias en la percepción de funcionalidad familiar según el grado de escolaridad de los padres, el tipo de familia y el sector de la ciudad, así: a) los adolescentes des padres con mayor nivel de escolaridad perciben mejor funcionalidad familiar, lo cual ha sido relacionado con mejor nivel económico, relaciones más igualitarias entre hombres y mujeres y relaciones más simétricas, basadas en el diálogo y la negociación (Palomar \& Márquez. 1999); b) los adolescente pertenecientes a familias nucleares perciben mejor funcionalidad, ello respalda los argumentos expuestos, en relación a que en estas familias los adolescentes encuentran mejor nivel socioeconómico, una parentalidad más afectiva, apoyo mutuo en las dificultades y mayor estabilidad emocional (UNICEF, 2003; Sánchez \& Valdés, 2011); c) la percepción de disfuncionalidad familiar fue muy variable entre los sectores de la ciudad, con diferencias que llegan hasta el 15\% entre una zona y otra. Esto sugiere que las medidas de atención primaria, prevención y promoción de las buenas relaciones familiares deben ser dirigidas reconociendo las particularidades de cada zona. Así, en el sector Nororiental, que presenta la mayor proporción de percepción de familias disfuncionales, deberían priorizarse acciones de atención primaria e intervenciones orientadas a contener esta problemática. Aún más si se tiene en cuenta que este hallazgo coincide con un estudio realizado en el año 2006 , que clasifica al $72 \%$ de las familias de esta zona como disfuncionales, y que indica altos índices de delito sexual y violencia intrafamiliar (Alcaldía de Medellín, 2012). Las acciones en esta zona podrían ser mediante apoyo familiar domiciliario dado por trabajadores sociales y psicólogos, que traten las necesidades 
Pág 175

La ausencia de figura paterna se ha asociado con inestabilidad emocional de los adolescentes, dificultades para generar un proyecto de vida propio, deserción escolar, embarazos, distorsión en las jerarquías familiares y perturbaciones en el proceso de emancipación de la familia (Villalba, 2014; Vanegas, Barbosa, Alfonso, Delgado, \& Gutiérrez, 2011). básicas y las crisis de las familias. En la zona suroccidental, que presenta la menor proporción de percepción de disfuncionalidad familiar, se requeriría de acciones para mantener y fomentar aspectos relacionados que conforman el perfil hallado. Un buen ejemplo de las acciones a implementar en estas zonas son las "escuelas de padres", en las que a través de reuniones grupales y de baja frecuencia, se promueven habilidades educativas y comunicativas entre padres e hijos. Finalmente, en los demás sectores, incluidos los corregimientos, que presentaron proporciones de percepción de disfuncionalidad familiar intermedias, las acciones deberían tener un énfasis preventivo, fomentando habilidades de comunicación, técnicas de solución de problemas, habilidades educativas y estrategias de afrontamiento frente a los conflictos familiares.

Es importante resaltar que el diseño de las intervenciones dirigidas a reducir la problemática de la disfunción familiar tiene en cuenta la presencia de factores de riesgo estructurales, como la composición y estatus de la familia, la monoparentalidad, el tamaño de las familias y aspectos socioeconómicos que son de difícil modificación, que se encuentran fuera del alcance de los programas de intervención; y factores funcionales o relacionales que se refieren a aspectos vinculados con el cuidado de los hijos, el conocimiento y uso de recursos sociales, la cohesión familiar, las pautas de comunicación, el modelo de conductas y las habilidades educativas, todos ellos susceptibles de modificación, por lo que son el centro de las estrategias descritas (Secades, Fernández, García, \& Al-Halabi, 2011).

Con respecto a la tipología de las familias de los adolescentes participantes de esta investigación, se destaca que el 45,5\% son familias monoparentales, principalmente con ausencia del padre, porcentaje superior al 15\% descrito en el 2002 por el Observatorio para la equidad y la integración social en Medellín y Antioquia (2006). Esta situación es relevante en tanto que la ausencia de figura paterna se ha asociado con inestabilidad emocional de los adolescentes, dificultades para generar un proyecto de vida propio, deserción escolar, embarazos, distorsión en las jerarquías familiares y perturbaciones en el proceso de emancipación de la familia (Villalba, 2014; Vanegas, Barbosa, Alfonso, Delgado, \& Gutiérrez, 2011).

Lo anterior no implica que las familias nucleares sean invariablemente mejores para el desarrollo de los adolescentes, ya que la tipología familiar explica sólo una parte en ese proceso de desarrollo. No obstante, sí sustenta que los adolescentes que crecen con el acompañamiento de ambos padres tienen un mejor entorno para el desarrollo de las habilidades sociales, cognitivas y emocionales (UNICEF, 2003; Vanegas et al., 2011). En este sentido, sería interesante observar los cambios en el desarrollo del adolescente, que se presentan en las configuraciones familiares constituidas por madres solteras. Por otro lado, en este estudio las familias monoparentales con presencia del padre y ausencia de la madre representaron el 8,6\%. Sobre este aspecto, en la literatura son exiguas las reflexiones acerca de los padres que asumen solos el cuidado de su progenie (Barrón, 2014).

La convivencia en familias extensas constituye una estrategia de supervivencia en las ciudades, ante las crisis económicas y los procesos de migración. Las viviendas son compartidas con otras personas como abuelos y tíos, lo que repercute en la dinámica familiar. En este tipo de familias, son comunes los conflictos intergeneracionales, particularmente entre abuelos y adolescentes, derivados de las diferencias entre las pautas de crianza drásticas, autocráticas y violentas que recibieron los adultos mayores y las resistencias que tienen para aceptar los valores democráticos 
que se recomiendan en la actualidad como el camino adecuado para formar a las nuevas generaciones (Puyana, 2004).

En referencia a la escala APGAR, se encontró que es un instrumento válido y fiable para dar cuenta de la percepción de funcionalidad familiar, que explica un porcentaje importante de la varianza, y que es de aplicación ágil. Lo anterior indica que se dispone de un instrumento útil que puede utilizarse en estudios descriptivos o analíticos, con muestras numerosas y en poblaciones heterogéneas, para evaluar y monitorear los programas familiares de intervención y prevención.

Las limitaciones de este estudio se encuentran en la falta de evaluación de las rutas de entrada a la monoparentalidad, las situaciones que las han originado y la duración o cronología de los trayectos monoparentales y su relación con el funcionamiento familiar. Asimismo, las asociaciones descritas presentan implicaciones para la formulación de hipótesis, más no para la atribución de causalidad. Por otro lado, es importante resaltar que en caso de que se pretenda hacer un diagnóstico clínico de las familias disfuncionales, la tamización del APGAR debería complementarse con otro tipo de evaluaciones, como un diagnóstico realizado por profesionales de trabajo social o psicología.

A pesar de las limitaciones del estudio, se halló una elevada prevalencia de percepción de disfuncionalidad familiar en la ciudad, se identificaron los grupos de adolescentes con percepción de mejor funcionalidad y los sectores de la ciudad con familias que requieren un diagnóstico profesional. Por otro lado, se puso en evidencia la disponibilidad de una escala válida y fiable para tamizar esta problemática, monitorizar su evolución en estudios posteriores y en general planear y evaluar intervenciones en la salud del adolescente, incluyendo los determinantes familiares.

\section{Referencias}

Alcaldía de Medellín. (2009). Protocolo de acompañamiento familiar. Recuperado de https://www.medellin.gov.co/irj/go/km/docs/wpccontent/Sites/Subportal\%20 del\%20Ciudadano/Medell\%C3\%ADn\%20solidaria/Secciones/Publicaciones/ Documentos/2011/Protocolo\%20familiar.pdf

Alcaldía de Medellín. (2012). Diagnóstico situacional de la infancia y adolescencia Medellín. Recuperado de https://www.medellin.gov.co/irj/go/km/docs/wpccontent/Sites/Subportal\%20del\%20Ciudadano/Bienestar\%20Social/Secciones/ Informes/Documentos/2012/Diagn\%C3\%B3stico\%20de\%20infancia\%20y\%20 adolescencia\%20nuevo\%20formato.pdf

Alcaldía de Medellín. (2014). Buen vivir en familia. Recuperado de: http://www.medellin.gov.co/iri/portal/ciudadanos?Navigation Target=navurl://b80c673c3db1c259b97f8c5422a4f4f5

American Academy of Pediatrics. (2003). Family pediatrics: Report of the Task Force on the Family. Pediatrics, 111(6), 1545-1571. https://www.ncbi.nlm.nih.gov/pubmed/12777595

Araujo, N. A., Macedo de Sousa, F., Silva, I., Oliveira, S. A., Macedo da Silva, D., \& Carvalho, S. E. (2012). Funcionalidade de familias de adolescentes de escolas públicas: uma abordagem desitiva. Cogitare Enferm, 17(2), 224-231. http://revistas. ces.edu.co/index.php/psicologia/article/view/3600/0

Ashby, T. \& Yaeger, A. M. (2003). Family Factors and Adolescent Substance Use: Models and Mechanisms. Current Directions in Psychological Science, 12(6), 222-226. http://cdp.sagepub.com/content/12/6/222.abstract 
Barrón, L. S. (2014). Familias monoparentales: un ejercicio de clarificación conceptual y sociológica. Revista del ministerio de trabajo y asuntos sociales 40 . Recuperado de http://www.isadoraduncan.es/es/book/export/html/361

Bell, L. G. \& Bell, D. C. (1982). Family Climate and the Role of the Female Adolescent: Determinants of Adolescent Functioning. Family Relations, 31(4), 519-527. http:// revistas.ces.edu.co/index.php/psicologia/article/view/3600

Berbesí, F. D., García, J. M., Segura, C. A., \& Posada, S. R. (2013). Evaluación de la dinámica familiar en familias de niños con diagnóstico de asma. Revista Colombiana de Psiquiatría, 42(1), 63-71. http://www.redalyc.org/pdf/806/80626357009.pdf

Cardona-Arias, J. A., Caro-Londoño, M., González, J. M., \& Franco-Mosquera, S. (2014). Construcción y evaluación de una escala sobre conocimientos en primeros auxilios en estudiantes de educación media Medellín-Bello. Revista CES Medicina, 28(1), 35-48. http://revistas.ces.edu.co/index.php/medicina/article/view/2732

Chaparro, S. W. \& Villalba, T. J. (2014). Responsabilidad de la función paterna y creencias para los adolescentes del área urbana del municipio de Sativá norte Boyacá. (Tesis de pregrado). Universidad Nacional Abierta y a Distancia, Bogotá, Colombia Recuperado de http://hdl.handle.net/10596/2503

Cuba, M., Jurado, A., Romero, Z., \& Cuba, M. (2013). Características familiares asociadas a la percepción de la calidad de vida en pobladores de un área urbano-marginal en el Distrito de Los Olivos, Lima. Revista Médica Herediana. 24, 12-16. http://www. upch.edu.pe/vrinve/dugic/revistas/index.php/RMH/article/view/728

Culpin, L., Heron, J., Araya, R., Melotti, R., \& Joinson, C. (2013). Father absence and depressive symptoms in adolescence: findings from a UK cohort. Psycholical medicine, 43 (12), 2615-2626. https://www.ncbi.nlm.nih.gov/pubmed/23673290

Gómez-Bustamante, E. M., Castillo-Ávila, I., \& Cogollo, Z. (2013). Predictores de disfunción familiar en adolescentes escolarizados. Revista Colombiana de Psiquiatría, 42(1), 72-80. http://www.elsevier.es/es-revista-revista-colombiana-psiquiatria379-avance-resumen-predictores-disfuncion-familiar-adolescentes-escolarizados-S0034745014600883

Gómez, C. F. \& Ponce, R. E. (2010). Una nueva propuesta para la interpretación de Family APGAR (versión en español). Atención familiar, 17(4), 102-106. http://new. medigraphic.com/cgi-bin/resumen.cgi?IDARTICULO=34451

González, S. F., Gimeno, C. A., Meléndez, M. J., \& Córdoba, I. A. (2012). La percepción de la funcionalidad familiar: Confirmación de su estructura bifactorial. Escritos de Psicología. 5(1), 34-39. http://scielo.isciii.es/scielo.php?script=sci abstract\&p $\mathrm{id}=\mathrm{S} 1989-8092012001100005$

Guibert, R. W. \& Torres, M. N., (2001). Intento suicida y funcionamiento familiar. Revista Cubana Medicina General Integral, 17(5), 452-60. http://scielo.sld.cu/scielo. php?script=sci arttext\&pid=S0864-21252001000500008

Moreno, J. \& Chauta, L. (2012). Funcionalidad familiar, conductas externalizadas y rendimiento académico en un grupo de adolescentes de la ciudad de Bogotá. Psychologia: avances de la disciplina, 6(1), 155-166. http://www.scielo.org.co/scielo.php?script=sci arttext\&pid=S1900-23862014000200004

Muyibi, A., Ajayi, I.-O., Irabor, A. E., \& Ladipo, M. M. A. (2010) A Relationship between adolescents' family function with sociodemographic characteristics and behaviour risk factors in a primary care facility. African Journal of Primary Health care family medicine, 2(1). doi: 10.4102/phcfm.v2i1.177

Negrete, C. A. \& Vite, S. A. (2011). Relación de la violencia familiar y la impulsividad en una muestra de adolescentes mexicanos. Acta Colombiana de Psicología, 14(2), 121-128. http://www.redalyc.org/html/798/79822611012/ 
Observatorio para la equidad y la integración social en Medellín y Antioquia. (2006). Viaje al interior de la familia, (15 Ed.). . Medellín: Comfama http://revistas.ces.edu. co/index.php/psicologia/article/view/3600

Palomar, L. J. \& Márquez, J. A. (1999). Relación entre la escolaridad y la percepción del funcionamiento familiar. Revista Mexicana de investigación educativa, 4(8), 299-343. http://revistas.ces.edu.co/index.php/psicologia/article/view/3600

Puyana, V. Y. (2004). La familia extensa: una estrategia local ante crisis sociales y económicas. Trabajo social, 6, 77-86. https://dialnet.unirioja.es/servlet/ articulo?codigo $=4389458$

Ramada-Rodilla, J. M., Serra-Pujadas, C., \& Delclós-Clanchet, G. L. (2013). Adaptación cultural y validación de cuestionarios de salud: revisión y recomendaciones metodológicas. Salud Pública de México, 55(1), 57-66. http://www.scielosp.org/ $\mathrm{pdf} / \mathrm{spm} / \mathrm{v} 55 \mathrm{n} 1 / \mathrm{v} 55 \mathrm{n} 1 \mathrm{a} 09 . \mathrm{pdf}$

Sánchez, E. P. \& Valdés, C. A. (2011). Una aproximación a la relación entre el rendimiento académico y la dinámica y estructura familiar en estudiantes de primaria. Revista Intercontinental de Psicología y Educación, 13(2), 177-196. http:// www.redalyc.org/pdf/802/80220774009.pdf

Secades, R. Fernández, J. R., García, G., \& Al-Halabi, S. (2011). Estrategias de intervención en el ámbito familiar. Barcelona: Diputació de Barcelona. http://www. prevencionbasadaenlaevidencia.net/uploads/PDF/EG Estrategiasintervencionfamiliar DIBA.pdf

Ulate-Gómez, D. (2013). Riesgo biopsicosocial y percepción de la función familiar de las personas adolescentes de sexto grado en la Escuela Jesús Jiménez. Acta méd costarric, 55(1), 18-23. http://bases.bireme.br/cgi-bin/wxislind.exe/iah/ online $/$ ? IsisScript=iah $/$ iah.$x$ is \&src=google\&base $=$ LILACS\&lang $=p \&$ nextAction $=1$ $\underline{n k \& \text { exprSearch }=700643 \& \text { indexSearch }=I D}$

Fondo de las Naciones Unidas para la Infancia (UNICEF), (2003). Nuevas formas de familia. Perspectivas nacionales e internacionales. Recuperado de http://www. unicef.org/uruguay/spanish/libro familia.pdf

Uparela, D. B., David, U.L., Reza, M. Y., Alzate, R. M., Carmona, H. Y., Zapata, V. N.. Rojas, J. F. (2011). Módulo dinámico familiar. Programa Medellín solidaria. Medellín: Alcaldía de Medellín

Vanegas, G., Barbosa, A., Alfonso, M., Delgado, L., \& Gutiérrez, J. (2011). Familias monoparentales con hijos adolescentes y psicoterapia sistémica: Una experiencia de intervención e investigación. Revista Vanguardia psicológica, 2(2), 203-215. https://dialnet.unirioja.es/descarga/articulo/4815139.pdf 